\title{
Performance of a rapid-scan vacuum Michelson interferometer at the NSLS
}

\author{
P. Brierley ${ }^{a)}$ \\ PIKE Technologies, 2901 Commerce Park Drive, Madison, Wisconsin 53717 \\ B. Badeau \\ Thermo Nicolet, 5225 Verona Road, Madison, Wisconsin 53711 \\ P. Dumas \\ LURE and LASIR-CNRS, Centre Universitaire Paris Sud, F-91405 Orsay, France
}

M. Smith ${ }^{\text {b) }}$

Thermo Nicolet, 5225 Verona Road, Madison, Wisconsin 53711

G. P. Williams ${ }^{\mathrm{c})}$

Jefferson Lab, 12000 Jefferson Avenue-MS 7A, Newport News, Virginia 23606

(Presented on 23 August 2001)

\begin{abstract}
A commercial Nicolet Magna-IR series rapid-scan Michelson Fourier transform infrared was installed in a vacuum housing and integrated into the U4IR beamline at the National Synchrotron Light Source (NSLS) at Brookhaven National Laboratory. The frequency reference laser was mounted outside the vacuum, but the moving mirror mechanism and the dynamic alignment system for the fixed mirror were in a vacuum. The performance of the instrument was measured in the usual way by measuring the repeatability of data collected under specific conditions of aperture, resolution, and mirror scanning velocity. We briefly discuss the beamline design, to put the interferometer in context, then present signal to noise data which we discuss in terms of both instrument performance and also storage ring beam stability. Under optimal conditions, the instrument has a reproducibility of $0.01 \%$ in $1 \mathrm{~min}$ of measuring time at a resolution of $2 \mathrm{~cm}^{-1}$, over a range from 100-3000 $\mathrm{cm}^{-1}$. (C) 2002 American Institute of Physics. [DOI: 10.1063/1.1425384]
\end{abstract}

\section{INTRODUCTION}

Infrared synchrotron radiation (IRSR) ${ }^{1-5}$ is significantly brighter than a thermal "globar" source but requires large extraction angles. In fact $1 \mathrm{~mA}$ of stored beam current in a storage ring has about the same brightness as a $2000 \mathrm{~K}$ black body. IRSR is also an absolute source, the brightness being determined by the stored beam current and the easy to calculate physics of the particular storage ring.

In the infrared one is confronted with the same issues as with other spectral ranges, namely spectral range and resolution, the mechanical manifestation, and the instrument performance. Since synchrotron radiation is broadband and since beamsplitting is straightforward, one naturally looks towards interferometers. These instruments have significant advantages over dispersive spectrometers-high throughput, multiplexing, and high resolution as illustrated in Fig. 1. Thus the anticipated performance of an interferometer is far superior to its dispersive counterpart.

The multiplexing advantage is only realized, however, if detector noise is the limiting factor, and one must therefore look at this issue carefully. In principle, synchrotron radiation is light emitted by the same electron bunches over and

\footnotetext{
a) Deceased.

${ }^{b}$ Present address, Thermo Elemental Corporation, 27 Forge Parkway, Franklin, MA 02038.

c)Electronic mail: gwyn@jlab.org
}

over again as they orbit the storage ring. The change in the number of particles is extremely small, and with up to $10^{12}$ particles per bunch, the shot noise is negligible. However, orbit instability is the major factor in generating effective noise in synchrotron radiation sources, and this usually defines the ultimate performance limit.

\section{BEAMLINE}

In previous articles, we have presented calculations of the brightness of synchrotron radiation and compared it with thermal sources. We have also described the U4IR beamline on which the present spectrometer was installed, but we summarize the essential characteristics here. The beamline extracts $90 \times 90$ milliradians of light from a 1.9 meter bending radius dipole on the $800 \mathrm{MeV}$ National Synchrotron Light Source (NSLS) visible ultraviolet storage ring. The light is reflected by a side-cooled plane mirror, and eventually brought to a 1:1 focus with an ellipsoidal mirror onto a wedged diamond window with a $10 \mathrm{~mm}$ clear aperture. After the diamond window, the diverging beam is collimated by a $430 \mathrm{~mm}$ focal length paraboloid, $5^{\circ}$ off axis. The collimated beam is then approximately $38 \times 38 \mathrm{~mm}$ with a diagonal of $53 \mathrm{~mm}$, and therefore slightly overfills the $40 \mathrm{~mm}$ optics of the Michelson interferometer. The emerging collimated beam from the Michelson was focused onto a detector using a 150 $\mathrm{mm}$ focal length, $90^{\circ}$ off-axis paraboloid for the measurements reported here. The liquid-helium cooled boron-doped 


\section{Conventional Grating} Instrument

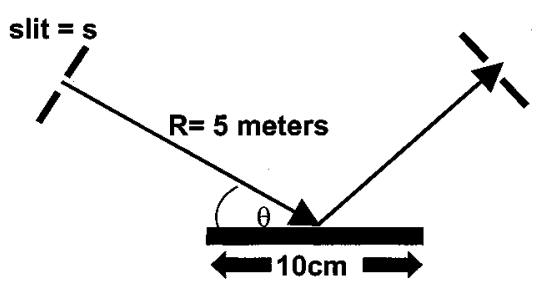

$\begin{aligned} & \text { Resolution given } \\ & \text { theoretically by: }\end{aligned} \frac{\lambda^{2}}{\Delta \lambda}=10 \mathrm{~cm}$

So, if $\lambda=50 A, \underline{\lambda / \Delta \lambda=2 \times 10^{7}}$

But, practically, if $\theta=2^{\circ}$, and if $s=10$ microns \# of resolution elements is $R \theta / 10=2 \times 10^{4}$

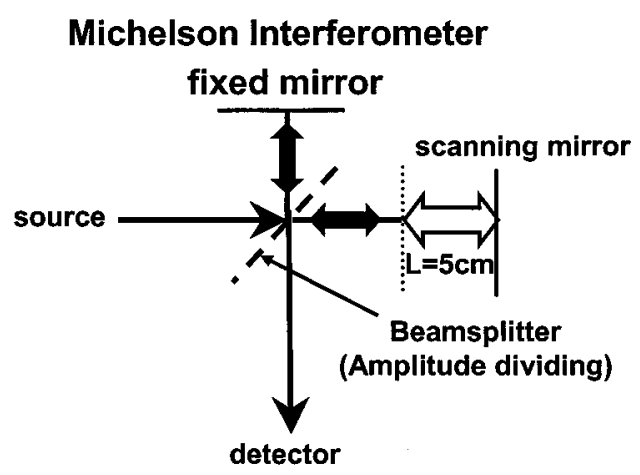

Resolution given theoretically AND practically by:

$$
\frac{\lambda^{2}}{\Delta \lambda}=10 \mathrm{~cm}
$$

Since $\lambda$ is in the range $1-1000$ microns, $\lambda / \Delta \lambda$ is in the range $10^{2}-10^{5}$
FIG. 1. Schematic of spectrometer and interferometer illustrating the fundamental differences between the two instruments and pointing out the practical advantages of the interferometer.
$2 \mathrm{~mm} \times 2 \mathrm{~mm}$ silicon bolometer detector from Infrared Laboratories was placed at the end of a $12 \mathrm{~mm}$ diameter Winston cone with an angular acceptance of $f / 4$. A $600 \mathrm{~cm}^{-1}$ low frequency pass filter cooled to $77 \mathrm{~K}$ was placed between the polyethylene window and the cone entrance. Note that the detector acceptance is much higher than the beam emittance. For example, in the diffraction limit the beam emittance is $\lambda^{2}$, and at $100 \mathrm{~cm}^{-1}$, the emittance is $0.01 \mathrm{~mm}^{2} \mathrm{sr}$, whereas the detector has an acceptance or etendue of $5.6 \mathrm{~mm}^{2} \mathrm{sr}$. For other experiments, the beam is focused to the sample using appropriate demagnifications prior to being refocused at the detector.

The beamline vacuum is as follows. Machine vacuum is preserved up to the diamond window. The collimating mirror and interferometer share a 50 mTorr vacuum, while a wedged polyethylene window separates this vacuum from the $<10^{-6}$ thermal vacuum of the detector. For some experiments additional windows were added between the spectrometer and the detector to allow samples to be contained at lower pressures.

Thus in the present configuration the Michelson was operated at a pressure of 50 mTorr, although we did pump the interferometer chamber to $10^{-5}$ Torr with a turbopump with no problems, and it seems likely that it could be used at this pressure. Going down to pressures of $<10^{-6}$ (thermal vacuum) would likely require the heat sinking of several components, but would not be too difficult.

\section{VACUUM MICHELSON INTERFEROMETER}

We preface this section by remarking that the motivation for the development of this instrument was the lack of availability of a commercial product with appropriate specifications. The commercially available instruments were either not true vacuum benches by virtue of using an air bearing, and/or contained instrumentation that was not necessary, such as sample compartments and detector optics. We were seeking a beamline "component" only. The specifications were as follows: ability to work in 50 mTorr pressure, 0.125 $\mathrm{cm}^{-1}$ best resolution, range from $10-5000 \mathrm{~cm}^{-1}$, with the range $10-2500 \mathrm{~cm}^{-1}$ covered by a single beamsplitter, rapidscan type instrument with scanning speed variable from 10 to $100 \mathrm{kHz}$ HeNe laser scan frequency, and $40 \mathrm{~mm}$ optics.

The principle of operation of this rapid-scan type of instrument is shown in Fig. 2. The beam is amplitude divided into two beams, one of which traverses a fixed path length, while the other traverses a path length which varies. In the normal mode of operation of such an instrument, the signal is measured for different and measured positions of the moving mirror. This is called step scanning. In order to be able to

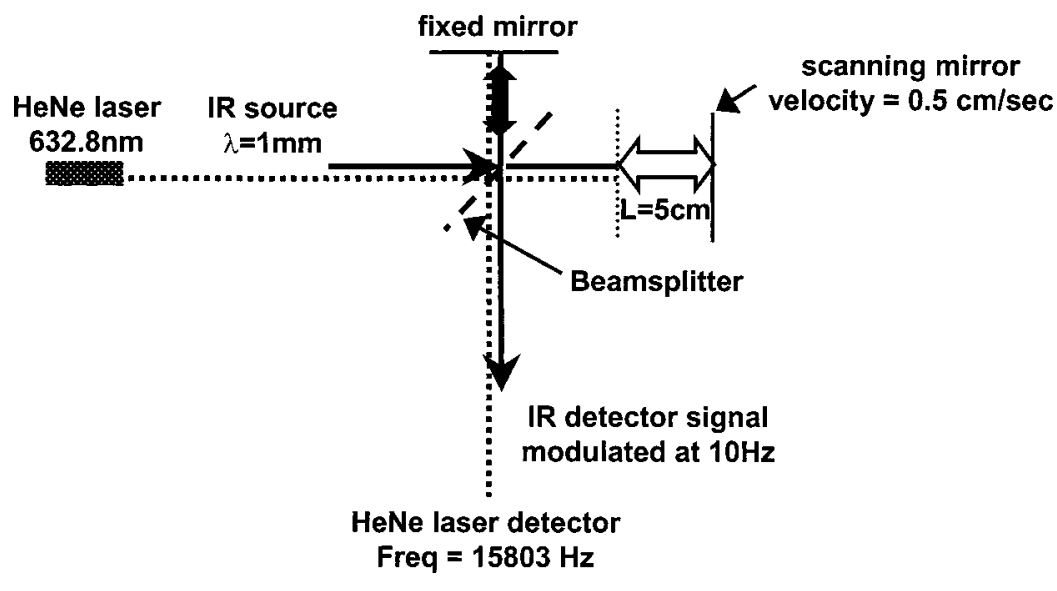

FIG. 2. Schematic of the operation of the rapid-scan Michelson interferometer, showing the built-in laser calibration. The elimination of a chopper means that one is always measuring all the light. 


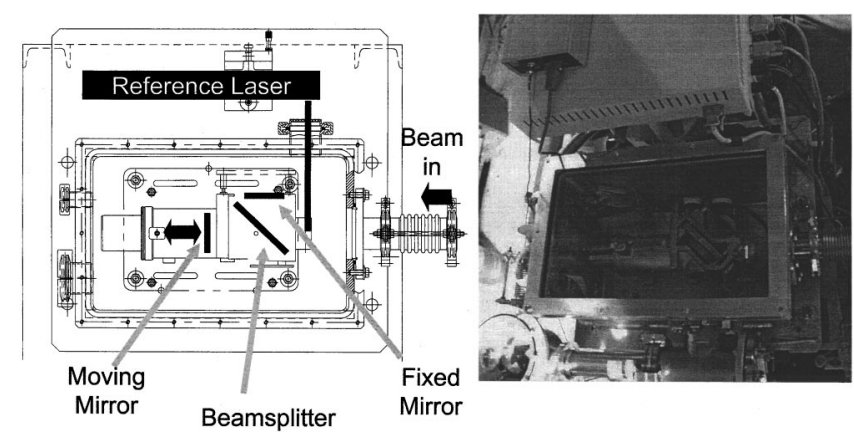

FIG. 3. Plan view and photograph of the vacuum Michelson interferometer.

detect a signal, the incoming beam is modulated using a chopper, and a lock-in amplifier is used to detect the synchronous signal from the detector. In a rapid-scan instrument, the moving mirror is moved at a constant velocity. This causes each input wavelength to be modulated at a specific frequency and has the advantage that no chopper is required so that one is looking at the signal all the time. Typically, one adjusts scanning speeds so that wavelengths being modulated are in the few 100 to few $1000 \mathrm{~Hz}$ region. The top frequencies depend on the detector response, while the lower frequencies should be high enough to be above laboratory noise whose main component is $60 \mathrm{~Hz}$. For accurate calibration purposes most such benches simultaneously modulate a laser beam as shown. The speed of the moving mirror is often specified by the modulation frequency of the HeNe laser. Since this laser has a wavelength of $632.8 \mathrm{~nm}$, if the mirror moves by $0.5 \mathrm{~cm} / \mathrm{s}$, so that the optical path difference is changing by $1 \mathrm{~cm} / \mathrm{s}$, then the laser modulation frequency will be $15803 \mathrm{~Hz}$. In addition, the resolution of the scan is proportional to the maximum path difference, while the highest frequency in the spectrum is proportional to the frequency of data taking during the scan.

The philosophy of the present application was to try to use commercial products if possible, and we selected the Nicolet Magna-IR (now Nexus) series of instruments. We purchased the Michelson interferometer, the power supply, reference laser, and electronics and repackaged them into a welded aluminum vacuum chamber that we designed ourselves. This instrument uses a graphite-in-glass bearing for the moving mirror, and previous tests showed that such a bearing works well in a vacuum. We also previously showed that the remainder of the electronics would function in a

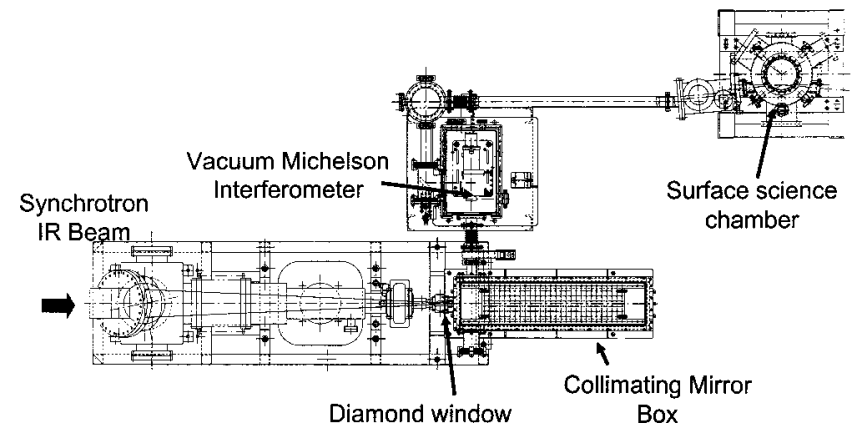

FIG. 4. Schematic of the beamline U4IR at the NSLS, showing the location of the vacuum Michelson interferometer.

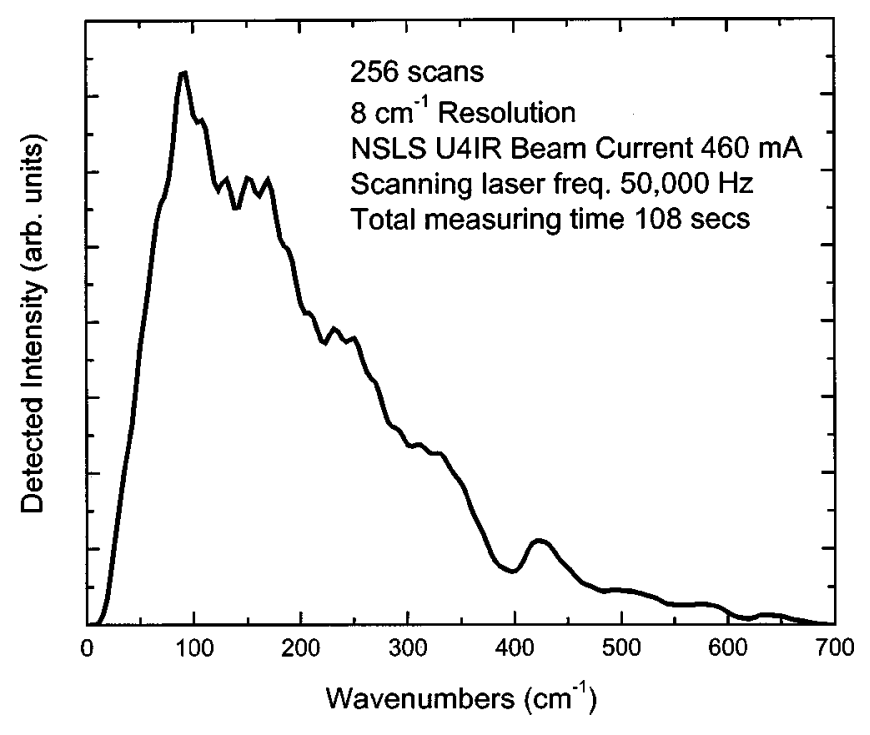

FIG. 5. Spectrum obtained using infrared synchrotron radiation and the Nicolet solid substrate beamsplitter. The low energy cutoff is believed to be due to diffraction, while the slow cutoff to higher frequencies is due to the black polyethylene filter in the detector.

vacuum. The result of the packaging of the instrument in a vacuum is shown in Fig. 3. The incorporation into the beamline is shown in Fig. 4.

After installation we tested the performance of the instrument by placing a detector directly after the instrument. Since the primary purpose of this beamline is low frequency surface science, which is brightness limited, the region from $50-800 \mathrm{~cm}^{-1}$ is of primary interest. In the performance tests we ran the bench at laser modulation frequencies from 10 to $120 \mathrm{kHz}$ and at various resolutions. There were no surprises. Clearly at the faster speeds, the mirror turnaround time represented a larger percentage of the total data gathering time, particularly at low resolution. At slower speeds, we observed laboratory noise. We note also that the interferometer is capable of scanning bidirectionally, but we did not utilize this

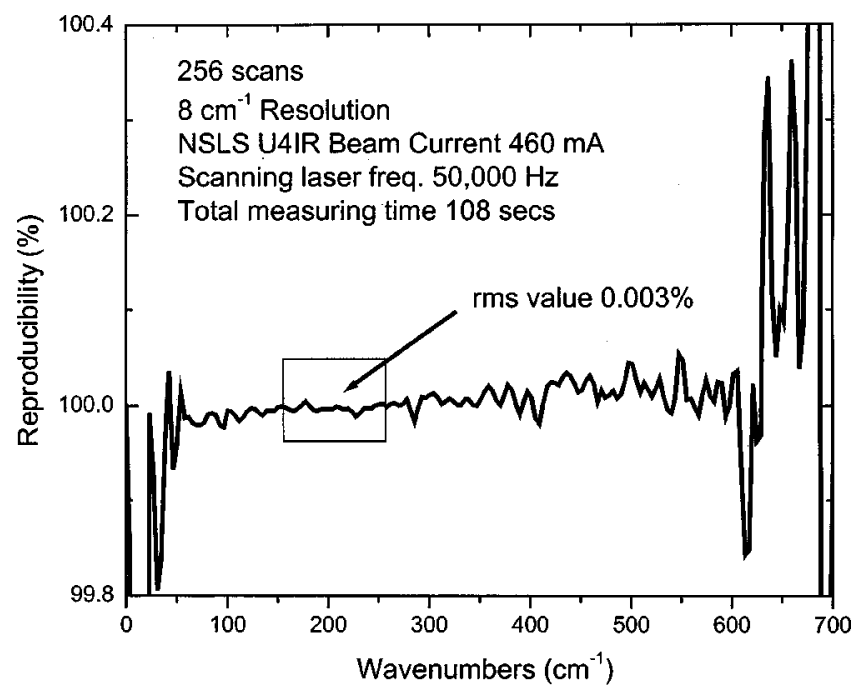

FIG. 6. Reproducibility of the vacuum Michelson interferometer in the range $0-700 \mathrm{~cm}^{-1}$ and showing that in the range $150-250 \mathrm{~cm}^{-1}$ root-meansquare deviations of only three parts in $10^{5}$ are obtained. Such values can be obtained up to $2500 \mathrm{~cm}^{-1}$ with suitable filtering with this beamsplitter. 
feature. The spectrum shown in Fig. 5 was selected to be under near optimum conditions. The "100\%" line showing the percentage ratio between two nominally identical scans is shown in Fig. 6. The cutoff to higher frequencies is due to the black polyethylene filter used in front of the bolometer. With other filters, we obtained spectra up to $2500 \mathrm{~cm}^{-1}$ with essentially the same performance as reported here.

\section{CONCLUSIONS}

The performance of the vacuum Michelson interferometer meets the requirements for the experiments. One can think of many improvements to the quality of life of the beamline, and we mention two of them here. First, it would be highly desirable to have a white-light source to be able to detect zero-path difference (when the two optical path lengths are identical) for calibration purposes. This can easily be done with the synchrotron beam, but since the beam is not always available it would be desirable to be able to do this independently of the synchrotron. The Nicolet interferometer does have such a white-light detector already mounted, and the implementation would not be difficult. Second, it would be useful if the mirror could collect data in both directions to minimize the loss of measuring time due to turnaround, a feature that is indeed becoming available in newer instruments.

\section{ACKNOWLEDGMENTS}

The authors are deeply grateful to many people who made this project possible. They acknowledge Ad Boyer and other staff of Thermo-Nicolet, Carol Hirschmugl and Mike Pilling of the University of Wisconsin-Milwaukee, and Larry Carr, Steve Kramer, Don Lynch, Gary Nintzel, Walter Stoeber, and B. Warasila of the NSLS. This work was supported by U.S. DOE Contract Nos. DE-AC05-84-ER40150 (JLab) and DE-AC02-98CH10886 (NSLS, BNL). The freeelectron laser is supported by the Office of Naval Research, the Commonwealth of Virginia, and the Laser Processing Consortium.

${ }^{1}$ W. D. Duncan and G. P. Williams, Appl. Opt. 22, 2914 (1983).

${ }^{2}$ G. P. Williams, Nucl. Instrum. Methods Phys. Res. A 291, 8 (1990).

${ }^{3}$ G. P. Williams, Rev. Sci. Instrum. 63, 1535 (1992).

${ }^{4}$ C. J. Hirschmugl and G. P. Williams, Rev. Sci. Instrum. 66, 1487 (1995).

${ }^{5}$ G. L. Carr, P. Dumas, C. J. Hirschmugl, and G. P. Williams, Nuovo Cimento D 20, 375 (1998). 